\title{
Diabetes mellitus as a compelling indication for use of renin angiotensin system blockers: systematic review and meta-analysis of randomized trials
}

In figure 3 the number of participants with myocardial infarction in the RAS blockers group of the STOP-2 trial is missing (BMJ 2016;352:i438, doi:10.1136/bmj.i438). The data for that group should show that 17 participants out of $235(17 / 235)$ had an outcome of myocardial infarction. 